\title{
Morphological diversity of panicle traits in Kam fragrant glutinous rice (Oryza sativa)
}

\author{
Qiyi Lei · Jiangju Zhou $\cdot$ Wenhua Zhang $\cdot$ Jing Luo $\cdot$ Kainian Wu \\ Chunlin Long $\mathbb{D}$
}

Received: 8 March 2017 / Accepted: 26 September 2017/Published online: 17 October 2017

(C) The Author(s) 2017. This article is an open access publication

\begin{abstract}
Panicle traits are the most intuitive and representative features of rice germplasm resources that have been utilized in the determination of its identity, genetic diversity, yield, and quality. Based on the approaches of ethnobotany, cultural anthropology and plant morphology, we analyzed the panicle traits of 95 traditional Kam fragrant glutinous rice landraces according to folk classification in Qiandongnan Prefecture of Guizhou Province in the past 10 years. A total of 95 Kam fragrant glutinous rice landraces were tested, of which $91 \%$ consisted of those with awns, and landraces with awn lengths of 2-6 cm comprised $62 \%$. The coefficient of variation (CV) of awn color, husk color and Grain color was more than $50 \%$, thereby indicating extensive morphological variations among the traditional Kam fragrant glutinous rice landraces. The average Shannon-Wiener value (diversity index, $\mathrm{H}^{\prime}$ ) for panicle qualitative traits was 0.50 , which was significantly higher than that of quantitative traits
\end{abstract}

Q. Lei · C. Long ( $ه)$

College of Life and Environmental Sciences, Minzu

University of China, Beijing 100081, China

e-mail: long.chunlin@muc.edu.cn

Q. Lei · J. Zhou - W. Zhang · J. Luo - K. Wu

College of Life and Environmental Sciences, Kaili

University, Guizhou 556000, China

C. Long

Kunming Institute of Botany, Chinese Academy of Sciences, Kunming 650201, China
(0.22), thereby indicating a relatively higher degree of genetic diversity among qualitative traits. Cluster analysis of panicle morphological traits showed that 95 Kam fragrant glutinous rice landraces could be divided into five categories, using the Euclidean distance of 0.68 as threshold. Each cultivar manifested unique panicle traits. The present study on the morphological diversity of Kam fragrant glutinous rice landraces provided basic information that may be utilized for the conservation and sustainable use of Kam fragrant glutinous rice landraces.

Keywords Rice panicle diversity - Germplasm resources · Dong ethnic people - Genetic diversity . Conservation

\section{Introduction}

Rice is a major food source for more than half of the world's population and has a major place in agricultural production. Rice germplasm diversity is an important genetic information transmission system (Nambara and Nonogaki 2012; Martínez-Andújar et al. 2012; Nachimuthu et al. 2015). It is not only the material basis and gene source of rice breeding and genetic improvement, but also the criterion in cultivating new rice landraces of high quality and adaptability. To investigate germplasm resources for crop utilization and improvement programs, it is essential to describe and evaluate the morphological 
characteristics of existing germplasm resources to effectively identify and differentiate each cultivar (Smith et al. 1991; Kumbhar et al. 2015; Ahmed et al. 2016). The panicle traits are the most intuitive and representative rice plant part that is utilized in determining its identity and genetic diversity, as well as in estimating rice yield and quality (Chen et al. 2001; Ma et al. 2006; Wu et al. 2016). Therefore, the characterization of rice panicle traits in relation to agricultural production and genetic diversity has long been an important research topic (Zhou et al. 2003; Wang et al. 2007; Zhou et al. 2014; Zhao et al. 2016; Adriani et al. 2016). As the study of genetic diversity of morphological traits can directly provide information on germplasm richness and provide important information for farmers or breeders especially when the resource population is large, using morphological traits to study genetic diversity is considered as a very simple and economical approach. Therefore, this classical method continues to be extensively employed in the evaluation of germplasm resources (Li et al. 2001; Hien et al. 2007; Veasey et al. 2008). At present, in evaluating crop germplasm resources, qualitative traits such as morphological features could be readily distinguished and identified, and quantitative traits have large differences (Horejsi and Staub 1999). Furthermore, morphological traits of germplasm resources are relatively more stable under various environmental conditions. Through scientific and effective sampling, using mathematical statistical methods on morphology traits of reproductive organs, several studies have evaluated the classification, relationships, and diversity of rice landraces (Oppong-Sekyere et al. 2011; Kumbhar et al. 2015; Afiukw et al. 2016; Sorkheh et al. 2016; Li et al. 2016), which are then utilized in the protection and rational utilization of germplasm resources.

The Dong people call themselves Kam. The fragrant glutinous rice grown by the Dong people, therefore, has been named as Kam fragrant glutinous rice. It is a special group of rice landraces that has been historically selected by the Dong and Miao nationalities of Qiandongnan Prefecture of Guizhou Province and its neighboring areas in China, and its name is synonymous to very fragrant cooked rice. It belongs to fragrant glutinous japonica type and has strong adaptability and resistance to stress. Commonly called "special rice" by domestic and foreign scholars. This group is also cultivated in the Dong areas of Tongdao
County in Hunan Province, and Sanjiang, Longsheng, and other counties in Guangxi Region, but most are located in the Dong areas of Liping, Congjiang, and Rongjiang counties in Qiandongnan Prefecture (Chaudhary et al. 2001). "Fragrant glutinous rice" is a common term for the fragrant glutinous rice propagated by the Dong people and is referred to as Oux or Kgoux in the Dong language, which can be translated as Kam rice of China or Kam sweet glutinous rice (Stone 2008). Kam fragrant glutinous rice has been historically cultivated in the region, and is thus rich in local landraces. In 1980, 349 landraces of Kam fragrant glutinous rice were collected from major cultivation areas such as Liping, Congjiang, and Rongjiang counties (Wu 1981). Since 2005, our research group has continuously been conducting a comprehensive survey of Kam fragrant glutinous rice in the Dong areas of Qiandongnan Prefecture, allowing us to conclude that there are about $100 \mathrm{Kam}$ fragrant glutinous rice landraces in the region. Although it seems that the region is rich in germplasm resources of Kam fragrant glutinous rice, the current number of landraces is $70 \%$ lower than that of the last century. Although previous studies have collected Kam fragrant glutinous rice landraces, but the diversity of germplasm resources in some of these villages are relatively low (Yu et al. 2005; Wu et al. 2010; Ma et al. 2010), whereas other investigations focused on the cultivation history, ecology, and culture-related problems of Kam fragrant glutinous rice (Yan 2008; Zheng et al. 2010; Wu et al. 2010). However, studies that systematically examined various agronomic and qualitative traits of Kam fragrant glutinous rice germplasm resources are limited, and no investigations on highly variable panicle traits have been conducted to date. The present study evaluated the genetic diversity of Kam fragrant glutinous rice in Qiandongnan Prefecture by identifying and performing correlation and cluster analyses of panicle traits of 95 landraces, and the resulting information may be utilized in the effective protection and rational use of its germplasm resources.

\section{Materials and methods}

A total of 368 accessions of germplasm resources of Kam fragrant glutinous rice (Oryza sativa L.) tested were collected from 36 ethnic villages in Qiandongnan 


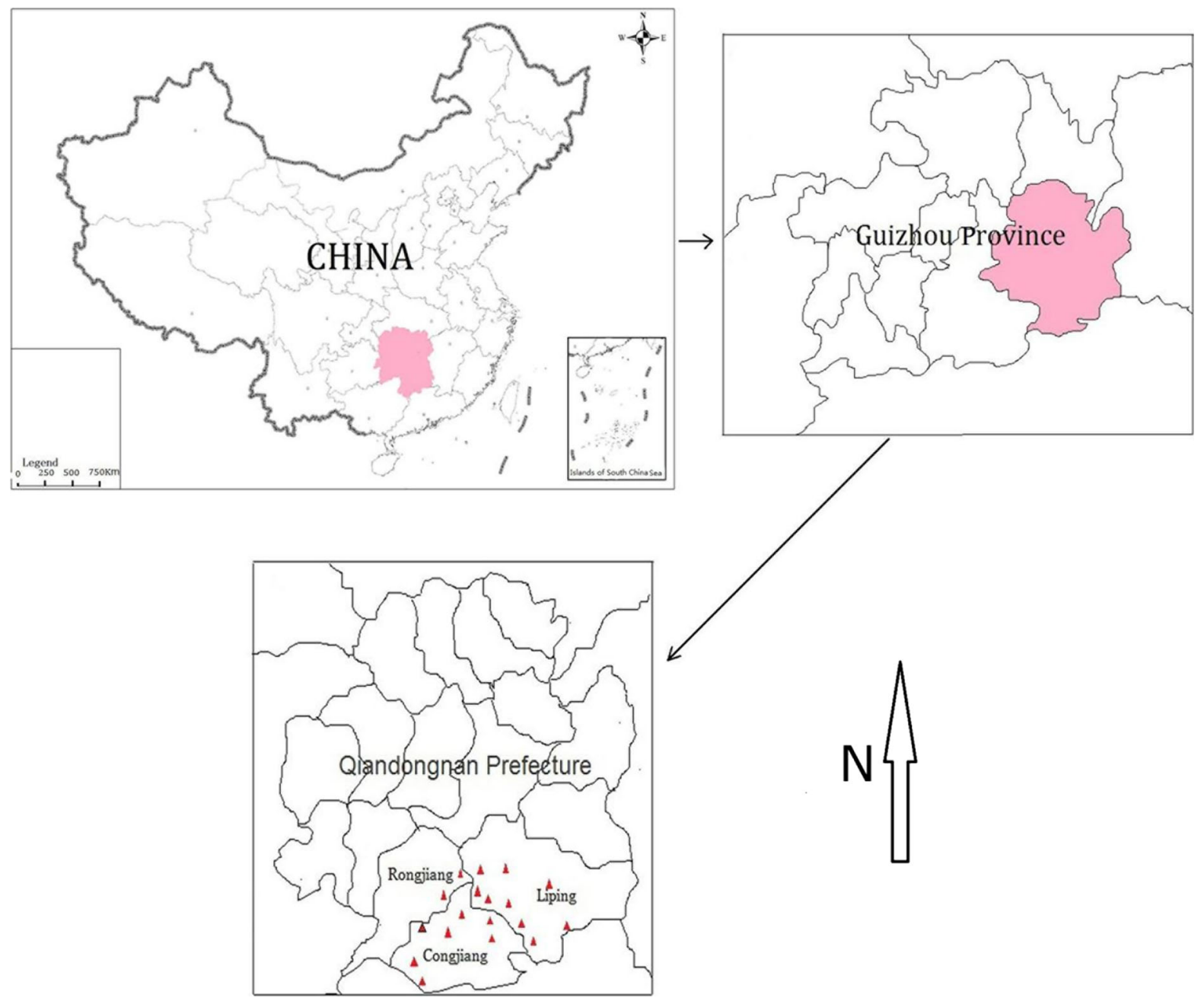

Fig. 1 The study area located in Qiandongnan Prefecture

Prefecture since 2005 (preserved in the Germplasm Bank of Kam Fragrant Glutinous Rice, Kaili University). During this period, we conducted a comprehensive survey and collection in the villages of Dong, Miao, Shui, and other ethnic groups at the junction of the Rongjiang, Liping, and Congjiang counties of Guizhou Province (Fig. 1), which are main producing areas of Kam fragrant glutinous rice in Qiandongnan Prefecture. Villagers in every village were required to preliminarily identify and record in detail the traits and names of all the landraces that they collected in the village and its adjacent areas (Chinese name and ethnic name). Finally, we invited a total of 26 villagers and village leaders (19 males and 7 females) in 13 Dong villages to identify and classify the germplasm resources of Kam fragrant glutinous rice collected in this area, and identified the remaining 95 Kam fragrant glutinous rice landraces as the experimental materials after separating different landraces with the same name or merging the same cultivar with different names.

The present study mainly used comprehensive integrated methods of ethnobotany, cultural anthropology, plant morphology, and other disciplines through access to relevant literature, field visits to investigate the production, life, social culture, and customs of the ethnic communities. Questionnaires, key informant interviews, participatory surveys, semistructured interviews, and other methods had been used to comprehensively collect data on Kam fragrant glutinous rice germplasm resources and related cultural information. 
Method of field test and the investigation of crop traits: According to different growing environments of different varieties, 95 varieties were planted in the experimental paddy fields of eight Dong villages (Huanggang, Yandong, Kengdong, Zhaoxing, Xiaohuang, Huanggang, Gaoqian, Bapa and Gaozeng) during 2015 and 2016. The seedling raising began on April 10th each year and then transplanted to the experimental plots on May 20th-30th. Randomized block design with three replications was used in the test. The planting row spacing was $26 \times 30 \mathrm{~cm}$. Each plant material was planted as three rows (10 plants each row). The management of water and fertilizer was used in the rice paddies through local traditional ways.

When the rice ripened, the main panicle was selected to test according to the method of Shen (1995). When harvesting, a total of 10-15 healthy main panicles of each variety were randomly selected for testing. Ten individuals of each variety were selected to measure the agronomic traits such as panicle length, grain number, 1000-grain weight, awn length and so on. Measuring tools included ruler, vernier caliper and electronic balance. The obtained data were calculated and got the average values of 2 years. The morphological characteristics of seeds were carefully observed and compared, which mainly included awn quality, awn color, awn length, grain shape, grain color, husk color, and endosperm color. Those results were recorded and photographed. The recorded characters, methods and criteria are shown in Table 1. The experimental data were processed by Excel 2007 and SPSS 11.0 software for statistical analysis.

We conducted detailed observation on panicle traits of $95 \mathrm{Kam}$ fragrant glutinous rice landraces referring to the "Rice Germplasm Resource Description Specification and Data Standard" (Han et al. 2006). The data analysis was carried out after assigning values to 6 qualitative traits of panicle. The panicle qualitative traits and their assigning values were shown in Table 2. When the germplasm was clustered according to the diversity index of morphological characters as well as the quality characters and quantitative characters, the hierarchical model of quantitative characters was adopted. That was the quantitative trait data were classified into 10 grades, 1 grade $<\mathrm{X}-2 \delta, 10$ grades $>\mathrm{X}+2 \delta$, each grade interval is $0.5 \delta$ between 1 and 10 grades, and $\delta$ is standard deviation. After standard quantification of 10 distinct traits of Kam fragrant glutinous rice panicles, statistical analysis of the average, standard deviation, maximum value, minimum value, range, and coefficient of variance was performed by using Excel and SPSS11.0 software. The Shannon-Weiner diversity index of each morphological trait was calculated by using the following formula: $\mathrm{H}^{\prime}=-\Sigma$ pi.lnpi. Finally, the genetic diversity of panicle traits was assessed by using the NTSYSpc 2.11 software, the SAHN program and UPGMA (Unwieghted Piar-group Method Arithmetic Averages) method was employed for cluster analysis, and a cluster diagram was constructed.

Table 1 The main morphological traits and their criteria for panicle characterization

\begin{tabular}{lll}
\hline Observed traits & Traits code & Recording standard \\
\hline Panicle length & PL & The length from the stem node of panicle to the tip of the panicle (cm) \\
Grains number & GN & Total grain number per panicle \\
1000-grain weight & GW & The weight of 1000 pure seeds in the dry air $(\mathrm{g})$ \\
Awn quality & AQ & Yes/no \\
Awn length & AL & The length from Husk top to awn tip $(\mathrm{cm})$ \\
Awn color & AC & White/black/black brown/red/red brown/brown/bronzing/yellow/tan \\
Grain shape & GS & Oval/rotundity/oblong \\
Husk color & HC & Blood red/red brown/black/black brown/yellow/tan/golden/dark/purple/brown/dark brown \\
Grain color & GC & Milk white/brown/bronzing/red/red brown/pale green/dark purple/purple red/brown \\
Endosperm color & EC & White/milky white \\
\hline
\end{tabular}


Table 2 Panicle qualitative traits and their given valuations

\begin{tabular}{ll}
\hline Qualitative traits & Given valuations \\
\hline Awn & Yes $=1$, no $=2$ \\
Awn color & White $=1$, black $=2$, red $=3$, brown $=4$, bronzing $=5$, yellow $=6$, tan $=7$ \\
Grain shape & Oval $=1$, rotundity $=2$, oblong $=3$ \\
Husk color & Red $=1$, brown $=2$, black $=3$, yellow $=4$, tan $=5$, golden $=6$, dark $=7$, purple $=8$ \\
Grain color & White $=1$, brown $=2$, bronzing $=3$, red $=4$, pale green $=5$, dark purple $=6$, purple red $=7$ \\
Endosperm color & White $=1$, milky white $=2$
\end{tabular}

\section{Results}

Morphological diversity of panicle traits of Kam fragrant glutinous rice landraces

The morphological traits of Kam fragrant glutinous rice caryopses and grains are highly heterogeneous (Fig. 2). The most prominent trait of Kam fragrant glutinous rice is a long awn (only a small number of landraces have no awn); the awns are rigid and the length of the awns are highly variable, the general length is within the range of $3-6 \mathrm{~cm}$, and the longest awn can be more than $12 \mathrm{~cm}$. Awn colors are also highly variable. There are more than 10 different colors, which include yellow, brown, red, and black.
Husk color is the second most important panicle morphological trait in Kam fragrant glutinous rice, with at least 10 colors known, including yellow, black, blood red, and brownish red. This morphological trait is also the most important basis for traditional classification and folk nomenclature. Grain color is the third major morphological trait in Kam fragrant glutinous rice, which includes milky white, red, black, brown, and purple black, and other colors, and this trait is also used in traditional classification and folk nomenclature. In addition, panicle length, seed bearing rate, grain length and width ratio, shape, endosperm color, and other traits are also highly variable in Kam fragrant glutinous rice.

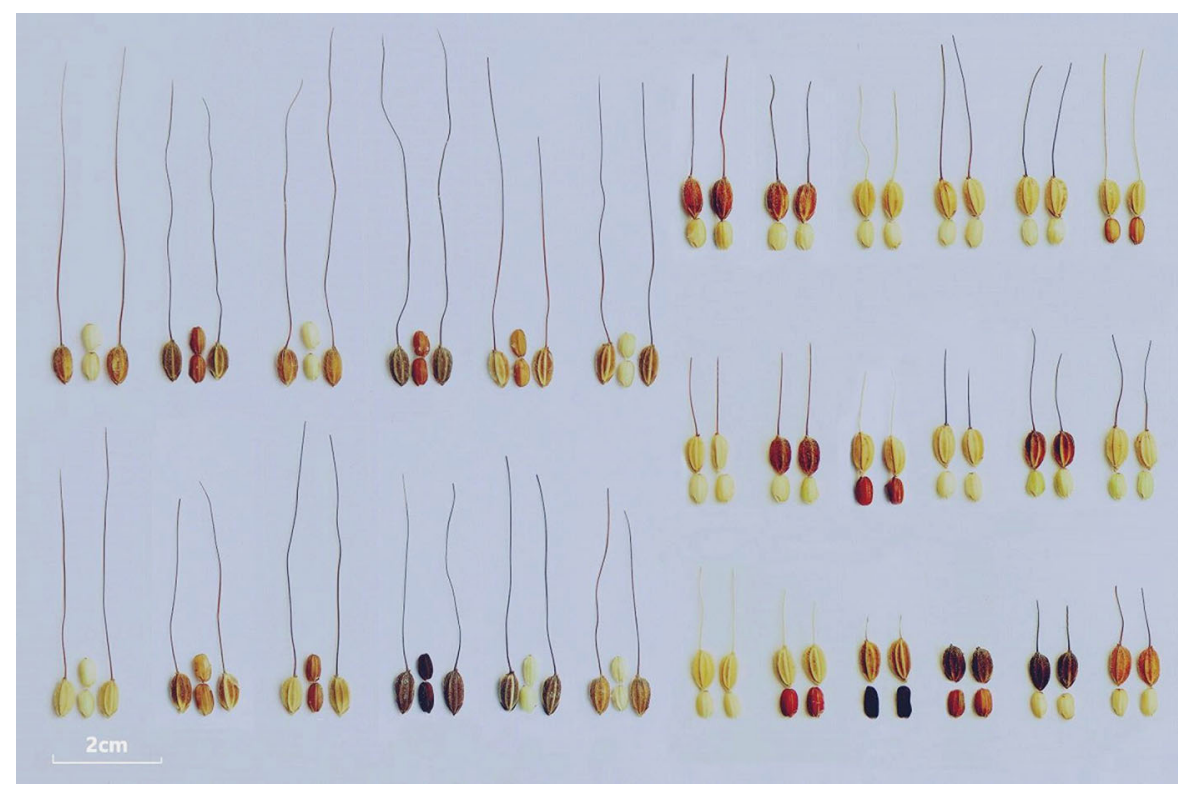

Fig. 2 Morphological diversity of caryopsis and grain traits in Kam fragrant glutinous rice 
Table 3 Variations in 10 panicle morphological traits of Kam fragrant glutinous rice

\begin{tabular}{lllllrrr}
\hline Serial number & Trait & Minimum & Maximum & Range & Average & SD & CV (\%) \\
\hline 1 & Panicle length & 20.15 & 38.27 & 18.12 & 26.53 & 3.11 & 11.72 \\
2 & Grains per panicle & 113 & 343 & 230 & 220.33 & 47.59 & 21.60 \\
3 & 1000-grain weight & 15.65 & 32.14 & 16.49 & 24.08 & 3.32 & 13.79 \\
4 & Awn & 1 & 2 & 1 & 1.50 & 0.50 & 33.33 \\
5 & Awn shape & 0 & 7.58 & 7.58 & 2.91 & 1.94 & 66.67 \\
6 & Awn color & 1 & 9 & 8 & 5.00 & 2.58 & 51.64 \\
7 & Grain shape & 1 & 3 & 2 & 2.00 & 0.82 & 40.82 \\
8 & Husk color & 1 & 11 & 10 & 6.00 & 3.16 & 52.70 \\
9 & Grain color & 1 & 9 & 8 & 5.00 & 2.58 & 51.60 \\
10 & Endosperm color & 1 & 2 & 1 & 1.50 & 0.50 & 33.33 \\
\hline
\end{tabular}

Coefficient of variation (CV) analysis of panicle morphological traits in Kam fragrant glutinous rice

By analyzing the average, standard deviation, and CV of 10 major and obvious panicle traits in Kam fragrant glutinous rice in this area, we show that there are extensive variations in panicle morphological traits in Kam fragrant glutinous rice (Table 3).

The CV of 10 main traits varied from 11.72 to $66.67 \%$, with awn shape showing the highest value $(66.67 \%)$. The other highly variable morphological features include husk color $(52.70 \%)$, awn color (51.64\%), and grain color (51.60\%), with CV values all $>50 \%$. The traits with lower degrees of variation included 1000-grain weight $(13.79 \%)$ and panicle length $(11.72 \%)$. The relatively higher $\mathrm{CV}$ in traits indicates that the traditional Kam fragrant glutinous rice landraces are highly variable in terms of morphological features.

Variation frequency analysis of panicle morphological traits in Kam fragrant glutinous rice

Statistical analysis of panicle traits of 95 landraces of Kam fragrant glutinous rice indicates a wide range of morphological differences, which in turn reflects the genetic diversity of the germplasm. Kam fragrant glutinous rice not only has significant differences in traits among the landraces, but also in the frequency of their occurrence (see Figs. 3, 4, 5, 6, and 7).

The most distinct morphological traits in Kam fragrant glutinous rice involved awn shape, awn color,

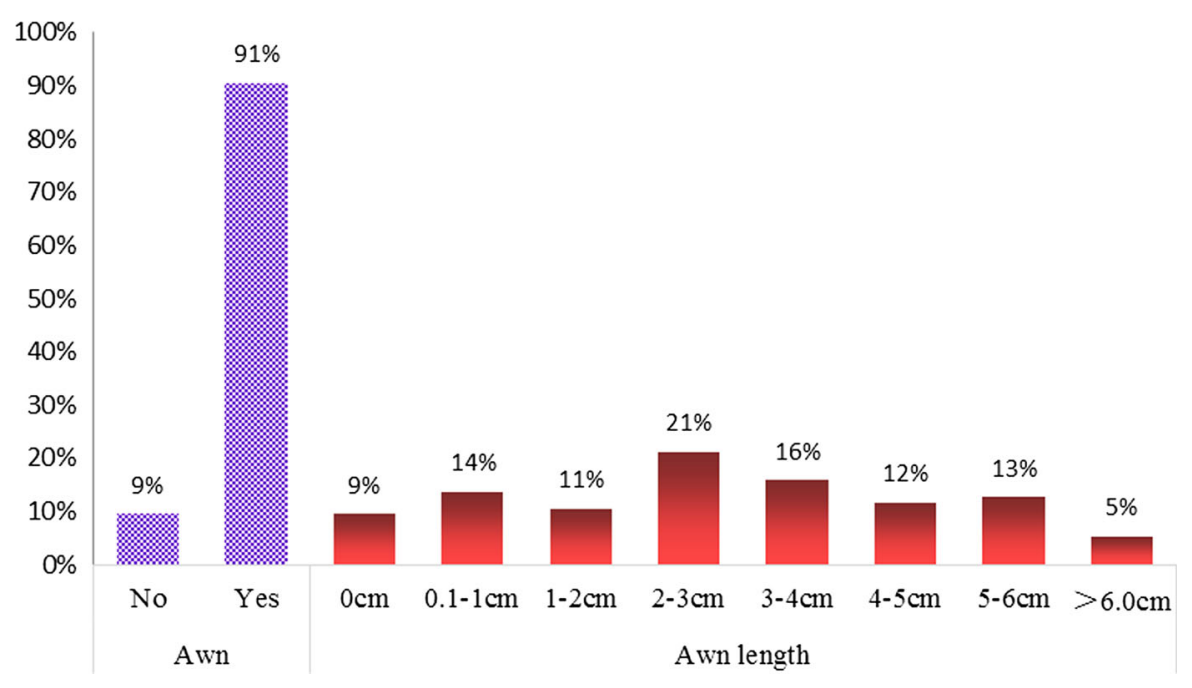

Fig. 3 Distribution frequency of Kam fragrant glutinous rice awn qualities 


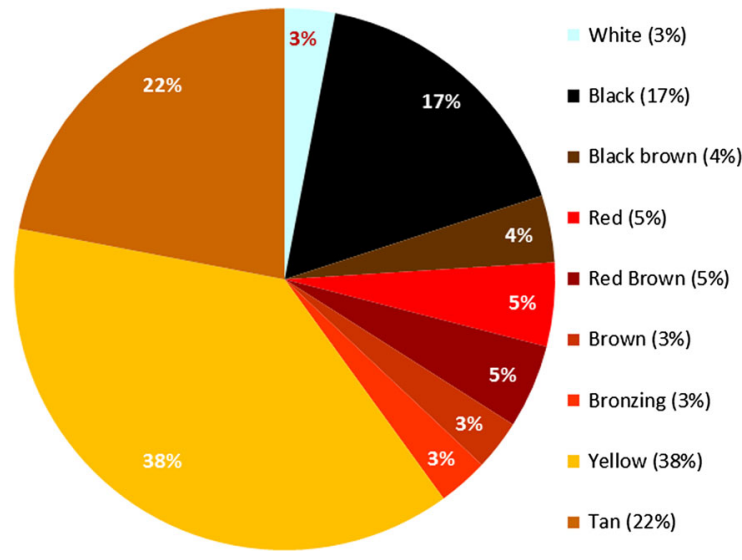

Fig. 4 Distribution frequency of awn colors

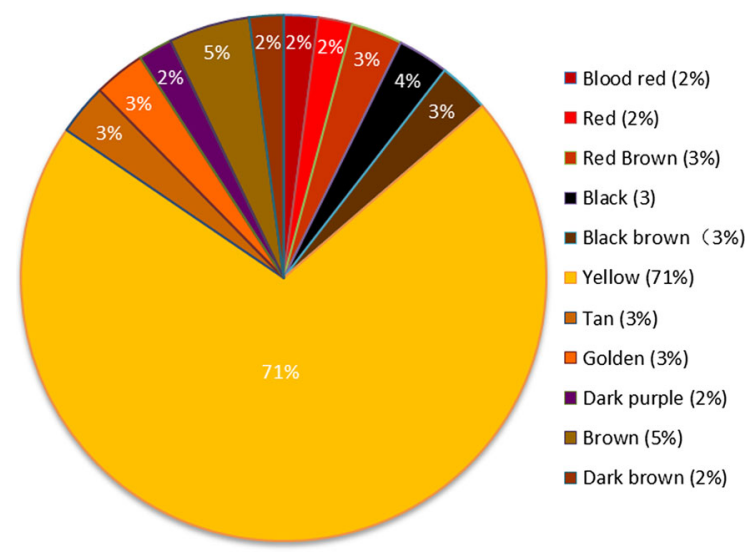

Fig. 5 Distribution frequency of husk colors

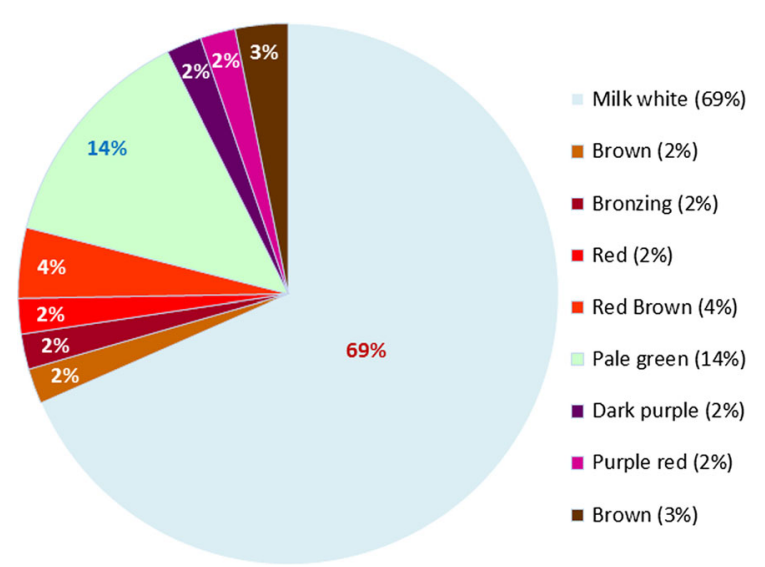

Fig. 6 Distribution frequency of grain colors

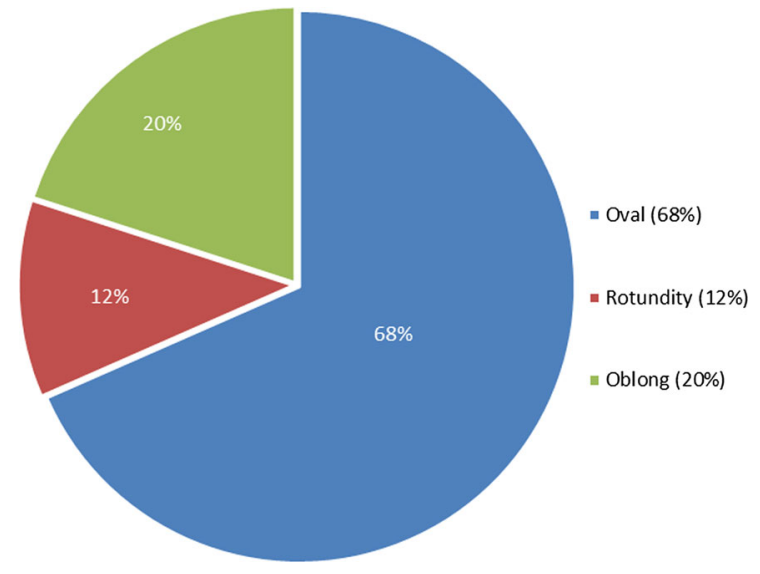

Fig. 7 Distribution frequency of grain shapes

husk color, and grain color. These traits are the main bases for distinguishing and identifying Kam fragrant glutinous rice germplasm resources. Among the 95 Kam fragrant glutinous rice landraces examined in the present study, 86 have a long awn with variable length (Fig. 3) and exhibited different colors (Fig. 4). Figure 3 shows that $91 \%$ of the Kam fragrant glutinous rice landraces have long awns, $62 \%$ have awn lengths within the range of 2-6 cm, and some landraces have awns with lengths $>10 \mathrm{~cm}$. There were nearly 10 awn colors, which were predominated by yellow (35\%) and brown (23\%), followed by black (15\%), red $(6 \%)$, brown $(3 \%)$, and brown-red $(3 \%)$. There were more than 10 husk colors (Fig. 5), which was predominated by yellow (72\%), followed by brown $(5 \%)$, black (2\%), and purple $(2 \%)$. In terms of grain color trait (Fig. 6), most landraces were milky white $(69 \%)$, followed by light green $(16 \%)$ and brown-red (4\%), black or purple-black (2\%), and red (2\%). Grain shapes generally consisted of three categories, namely, oval (68\%), long (20\%), and nearly round (12\%) (Fig. 7).

Shannon-Wiener diversity index of panicle morphological traits in Kam fragrant glutinous rice

The Shannon-Weiner diversity index $\left(\mathrm{H}_{\mathrm{S}}=-\Sigma\right.$ pi $\left.\cdot \operatorname{lnpi}\right)$ of each morphological trait was calculated after normalization of the results of panicle trait evaluation (Table 4). Among the four quantitative traits of Kam fragrant glutinous rice, the highest Shannon-Wiener diversity index was that of awn length (0.29). The diversity index of the total grain number per panicle 
was 0.24 , and that of the panicle length was 0.17 . The average diversity index of all quantitative traits was 0.22. Among the six qualitative traits, the highest Shannon-Wiener diversity index value was that of awn color (0.76), followed by husk color (0.63), whereas the lowest was awn quality (0.32). The average diversity index of all qualitative traits was 0.50 . As a whole, the Shannon-Wiener diversity index of qualitative traits was significantly higher than that of the quantitative traits, which was indicative of more extensive genetic diversity.

Cluster analysis of panicle morphological traits in the Kam fragrant glutinous rice germplasm

Cluster analysis indicated that the 95 Kam fragrant glutinous rice landraces could be divided into five categories, using the Euclidean distance of 0.68 as the threshold value (Fig. 8). The first category consisted of 14 landraces, which were characterized by a long awn, with the longest awn $>10 \mathrm{~cm}$ in length, the awn shape, husk color, and grain color showed the highest diversity, and most landraces were cultivated at relatively higher altitudes. The second category was the largest, consisting of a total of 71 landraces, which have relatively shorter awns, or in most landraces, only the top grains have relatively long awns, whereas other grains have shorter or no awns. Most of these landraces are cultivated at a low altitude. There were several earlymaturing landraces, which may be utilized for traditional crop rotation or mixed management. The third category included four landraces that possessed relatively shorter awns and yellow husks. The fourth category consisted of two landraces, namely, the Bazhou black rice and Diping black rice, which develop short or no awns and exhibit unique husk and grain colors. The Bazhou black rice is the most popular black fragrant glutinous rice in the region. The fifth category included four landraces, namely, Huangshanxue rice, red rice, bright red fragrant glutinous rice, and Shuiniumao rice, which presented relatively longer awns, higher yield, were mainly cultivated in the mediate altitude of the hillside, and were highly resistant to pests.

\section{Discussions}

The present study analyzed the panicle traits of 95 traditional Kam fragrant glutinous rice landraces, which showed that the Kam fragrant glutinous rice germplasm is highly diverse in terms of both qualitative and quantitative traits. Different types of Kam fragrant glutinous landraces manifest their own unique traits, and extensive variation in panicle traits was observed. Although morphological traits represent the results of the interaction between gene and the environment, these can, to a certain extent, reflect the degree of genetic variation and species diversity, which has long been an important basis for understanding the diversity of crop landraces. Because morphological traits are influenced by the interaction between genes and environmental factors, it is essential to comprehensively and accurately understand and evaluate these phenotypes in relation to the results of AFLP, SSR, or other molecular marker technique analysis. Because Kam fragrant glutinous rice is a unique rice variety in the Dong and Miao areas in Qiandongnan Prefecture, its diversity is not only related to the natural ecological environment, but also to the traditional culture of the region (Lei et al. 2013). For this reason, in the evaluation, protection, and utilization of Kam fragrant glutinous rice germplasm resources, we should fully consider the influence of ethnic culture on the formation and protection of Kam

Table 4 Shannon-Wiener diversity index of panicle morphological traits in Kam fragrant glutinous rice

\begin{tabular}{llll}
\hline Qualitative trait & Shannon-Wiener diversity index $\left(\mathrm{H}^{\prime}\right)$ & Quantitative trait & Shannon-Wiener diversity index $\left(\mathrm{H}^{\prime}\right)$ \\
\hline Awn & 0.32 & Panicle length & 0.21 \\
Awn color & 0.76 & Awn length & 0.29 \\
Grain shape & 0.34 & Total grains per panicle & 0.24 \\
Husk color & 0.63 & 1000 -grain weight & 0.17 \\
Grain color & 0.58 & & \\
Endosperm color & 0.37 & & 0.22 \\
Average & 0.50 & Average & \\
\hline
\end{tabular}




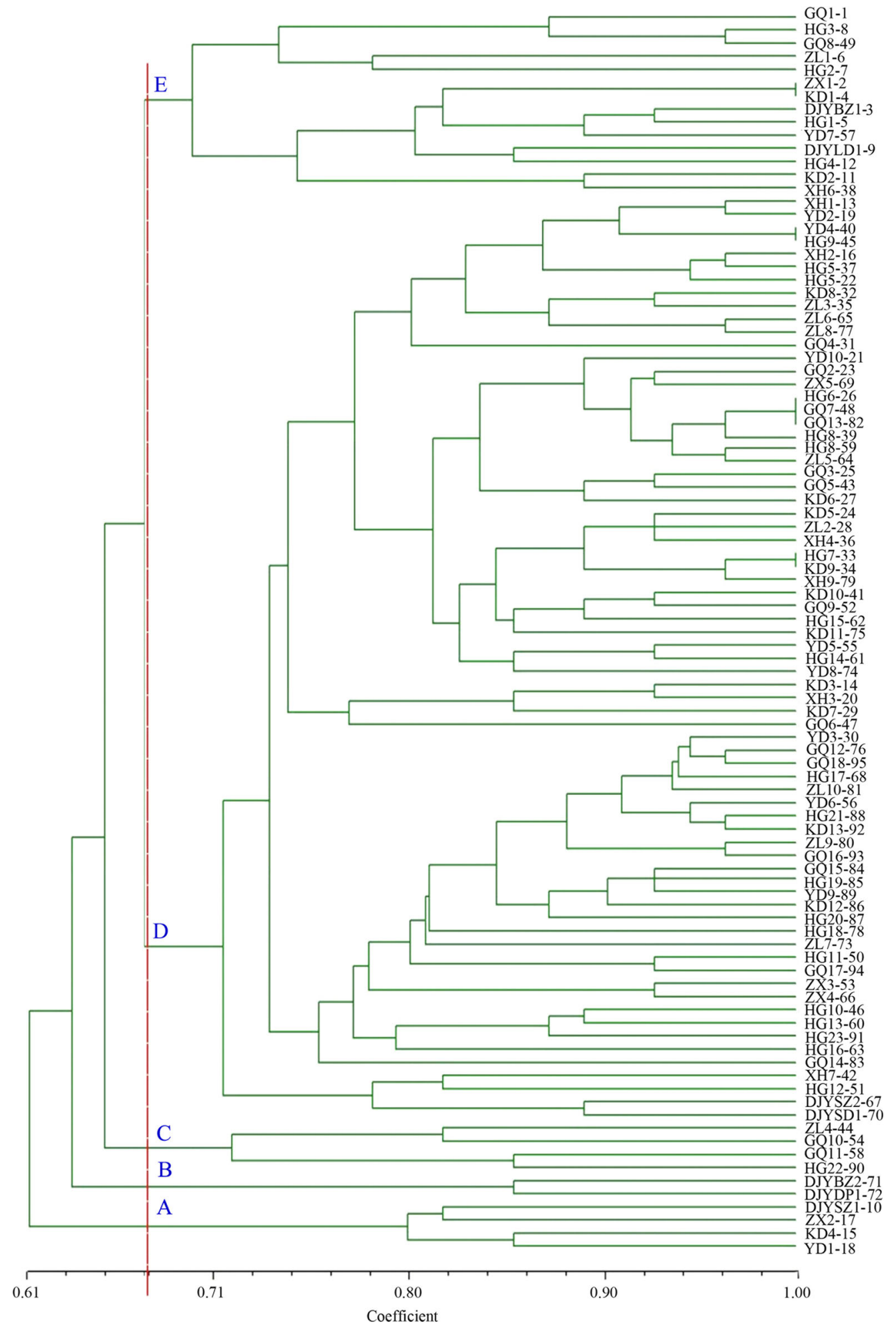

Fig. 8 UPGMA dendrogram of the panicle traits of 95 Kam fragrant glutinous rice landraces 
fragrant glutinous diversity and formulate effective measures and strategies for its protection and rational utilization.

Genetic diversity of panicle traits in Kam fragrant glutinous rice landraces

The diversity of Kam fragrant glutinous rice landraces is intuitively reflected in the diversity of their panicle traits. The awn exhibits important morphological traits in Kam fragrant glutinous rice. The colors of the awns were also rich and highly variable. In addition, the quantitative traits of awn length, panicle length, and grain shape also showed high genetic diversities. These important panicle morphological traits and differences in Kam fragrant glutinous rice were the most important bases for the diversified nomenclature of Kam fragrant glutinous rice landraces. According to these main panicle traits, the local middle-aged and elderly villagers of Dong and Miao do not need to observe plant height, plant type, first branch or secondary branches, and other (characteristic) traits as almost all of them could identify and distinguish a wide range of Kam fragrant glutinous rice landraces. For a long time, local Miao and Dong ethnic people have selected germplasm resources based on their living environment, social customs, traditional culture, and life needs, and have performed breeding based on panicle traits to maintain the diversity of the Kam fragrant glutinous rice germplasm. Investigations on the morphological diversity of traditional local crop landraces may thus improve our understanding of the richness of available germplasm resources, which may be utilized by rice breeders.

Mechanisms underlying the development of different panicle morphological traits in Kam fragrant glutinous rice landraces

Complexities in the geographical environment and the traditional culture of Qiandongnan Prefecture of Guizhou Province are the major forces that led to the current diversity in the Kam fragrant glutinous rice germplasm. The lowest altitude of this area is $147.8 \mathrm{~m}$ and the highest altitude is $2178.8 \mathrm{~m}$. The area contains many mountains and deep valleys; the mountains are cool and dry, whereas the valleys are humid and hot. The area has distinct stereoscopic climate and the vegetation is highly diverse. The Miao and Dong ethnic people live mainly in the mountainous areas. Their fragrant glutinous rice plants are cultivated at different altitudes, ranging from 200 to $1600 \mathrm{~m}$ above sea level and adapt well to these complex and varied natural environments, thereby forming a unique and rich ecological type such as the 60-day, 90-day, coldwater, and high-mountain rice landraces, and their phenotypes also show a wide range of traits, indicating that the observed morphological diversity is the result and comprehensive reflection of the interaction between genetic diversity and environmental diversity. Therefore, the diversity in Kam fragrant glutinous rice traits is not only related to the spectrum of features of the natural environment of the region, but is also closely related to the traditional culture of the region. The food, literature, and art culture, religious beliefs and folk activities, and other traditional cultures of the Miao and Dong ethnic groups cannot be separated from the application of Kam fragrant glutinous rice, and hundreds of traditional festivals within a year are closely associated with the use of Kam fragrant glutinous rice. Therefore, Kam fragrant glutinous rice plays a major role in the ethnic communities of the region, starting from their birth to their death (Lei et al. 2013). In particular, landraces with red, black, and purple grain colors are often used in festive, funeral, or regular ritual activities. Therefore, it is necessary to maintain the diversity of Kam fragrant glutinous rice landraces, and the diversity of ethnic cultures is a very important factor in maintaining the Kam fragrant glutinous rice diversity. In addition, for the Miao, Dong, and other nationalities, long-term production practices include Kam fragrant glutinous rice in plant rotation, comprehensive management, and other traditional management for pest control and yield improvement, and thus they actively maintain a rich Kam fragrant glutinous rice germplasm resources. Every household in the region generally grows 3-4 landraces every year, some farmers have as many as 7-8 landraces. The Kam fragrant glutinous rice landraces among households and villages have great heterogeneity, forming a diversity coexistence pattern of germplasm resources.

Conservation of Kam fragrant glutinous rice germplasm diversity

Panicle diversity in Kam fragrant glutinous rice reflects the spectrum of characteristics of the 
germplasm, which was influenced by the interaction between the natural environment and multi-ethnic traditional cultural needs. Kam fragrant glutinous rice is thus a highly valuable rice variety. However, since 2008-2015, extensive efforts in urbanization, which included the development of aviation runways, expressways, high-speed railways, and rural highways, destruction of several Chinese fir forests, and widespread application of pesticides, have resulted in the deterioration of the environment. The cultivation areas of Kam fragrant glutinous rice landraces were largely reduced, and germplasm diversity rapidly decreased each year. Recently, due to rapid economic development and the needs of ethnic poverty-stricken areas in the region, mountain farmers have opted to serve as migrant workers, and the traditional culture of the ethnic minorities was then mixed with that of mainstream culture. The traditional Kam fragrant glutinous rice farming culture and traditional ethnic culture are slowly getting lost. The diversity of the Kam fragrant glutinous rice germplasm has thus been fundamentally threatened. Therefore, it is imperative to conduct a comprehensive evaluation of Kam fragrant glutinous rice germplasm diversity, combined with the needs for socio-economic and traditional ethnic culture development in the region, develop dynamic protection methods that are in line with the traditions of Kam fragrant glutinous rice farmers in the region, and to implement measures to protect the natural ecological and traditional ethnic cultural environments to maintain the Kam fragrant glutinous rice germplasm.

Acknowledgements This study was supported by the National Natural Science Foundation of China (31360070, 31660083 and 31761143001), the Ministry of Science and Technology (2012FY110300), the Natural Science Foundation of Guizhou Province (2010-2078), the Natural Science United Foundation of the Guizhou Province (LKK201305), the Science Foundation of Department of Education of the Guizhou Province (20090082), and the Innovation Team of Conservation of Genetic Resources and Associated Traditional Knowledge of Department of Education of Guizhou Province.

Open Access This article is distributed under the terms of the Creative Commons Attribution 4.0 International License (http:// creativecommons.org/licenses/by/4.0/), which permits unrestricted use, distribution, and reproduction in any medium, provided you give appropriate credit to the original author(s) and the source, provide a link to the Creative Commons license, and indicate if changes were made.

\section{References}

Adriani DE, Dingkuhn M, Dardou A, Adam H, Luquet D, Lafarge T (2016) Rice panicle plasticity in Near Isogenic Lines carrying a QTL for larger panicle is genotype and environment dependent. Rice 9(1):28

Afiukw CA, Faluyi JO, Atkinson CJ, Ubi BE, Igwe DO, Akinwale RO (2016) Screening of some rice varieties and landraces cultivated in Nigeria for drought tolerance based on phenotypic traits and their association with SSR polymorphism. Afr J Agric Res 11(29):2599-2615

Ahmed MS, Bashar MK, Shamsuddin AKM (2016) Diversity level, Spearman's ranking and core collections from 98 rice germplasm through quantitative, qualitative and molecular characterizations. Rice Genomics Genet 7(2): $1-10$

Chaudhary RC, Tran DV, Duffy R (2001) Specialty rices of the world: breeding, production and marketing. Food and Agriculture Organization of the United Nations (FAO), Rome, pp 1-20

Chen WF, Xu ZJ, Zhang WZ, Zhang LB, Yang SR (2001) Creation of new plant type and breeding rice for super high yield. Acta Agron Sin 27(5):665-672

Han LZ, Wei XH, Chao GL (2006) Specification and data standard for rice germplasm resources. China Agricultural Press, Beijing

Hien NL, Sarhadi WA, Oikawa Y, Hirata Y (2007) Genetic diversity of morphological responses and the relationships among Asia aromatic rice (Oryza sativa L.) landraces. Tropics 16(4):343-355

Horejsi T, Staub JE (1999) Genetic variation in cucumber (Cucumis sativus L.) as assessed by random amplified polymorphic DNA1. Genet Resour Crop Evol 46(4):337-350

Kumbhar SD, Kulwal PL, Patil JV, Sarawate CD, Gaikwad AP, Jadhav AS (2015) Genetic diversity and population structure in landraces and improved rice varieties from India. Rice Sci 22(3):99-107

Lei QY, Zhang WH, Sun J, Yang MX, Zhou JJ (2013) Traditional management and utilization of glutinous rice genetic resources in Southeast Guizhou. Plant Divers Resour 35(2):195-201

Li Z, Zhang H, Zeng YW, Shen SQ, Sun CQ, Wang XK (2001) Studies on phenotypic diversity of rice germplasm in Yunnan, China. Acta Agron Sin 27(6):832-837

Li ZJ, Ma SS, Bu LQ, Li YD, Tang H, Zhang YX, Tian L, Li PF (2016) Analysis of genetic diversity of phenotypic traits of rice germplasm resources that introduced in Ningxia. Jiangsu Agric Sci 44(8):117-121

Ma Z, Huo EW, Lu ZC, Xu SK (2006) Effect of main characters on yield of hybrid rice. Shandong Agric Sci 3:21-23

Ma L, Yu XQ, Zhao FS (2010) Establishment of SSR finger print map of local rice varieties He' in Guizhou, Southwest China. J Agric Sci 23(1):5-10

Martínez-Andújar C, Martin RC, Nonogaki H (2012) Seed traits and genes important for translational biology-highlights from recent discoveries. Plant Cell Physiol 53(1):5-15

Nachimuthu VV, Muthurajan R, Duraialaguraja S, Sivakami R, Pandian BA, Ponniah G, Sabariappan R (2015) Analysis of population structure and genetic diversity in rice 
germplasm using SSR markers: an initiative towards association mapping of agronomic traits in Oryza sativa. Rice 8(1):30

Nambara E, Nonogaki H (2012) Seed biology in the 21st century: perspectives and new directions. Plant Cell Physiol 53(1): $1-4$

Oppong-Sekyere D, Akromah R, Nyamah EY, Brenya E, Yeboah S (2011) Characterization of okra (Abelmoschus spp.) germplasm based on morphological characters in Ghana. J Plant Breed Crop Sci 3(13):368-379

Shen ZT (1995) Crop breeding experiment. China Agriculture Press, Beijing, pp 102-107

Smith SE, Al-Doss A, Warburton M (1991) Morphological and agronomic variation in North African and Arabian alfalfas. Crop Sci 31(5):1159-1163

Sorkheh K, Masaeli M, Chaleshtori MH, Adugna A, Ercisli S (2016) AFLP-based analysis of genetic diversity, population structure, and relationships with agronomic traits in rice germplasm from North region of Iran and world core germplasm set. Biochem Genet 54(2):177-193

Stone R (2008) Chinese province crafts pioneering law to thwart biopiracy. Science 320(5877):732-733

Veasey EA, Silva EF, Schammass EA, Oliveira GCX, Ando A (2008) Morphoagronomic genetic diversity in American wild rice species. Braz Arch Biol Technol 51(1):94-104

Wang CH, Xu ZJ, Xia YS, Zhang YZ, Zhen WJ, Wang H, Zhao JM (2007) Analysis on correlation between rice panicle traits and yield. Liaoning Agric Sci 3:54-56

Wu XH (1981) Investigation report of "He" in Liping, Congjiang and Rongjiang county. Guizhou Agric Sci $5: 65-70+64$
Wu YH, Pu XC, Yang CY, Long YX (2010) Classification of special rice varieties and its leaf-age cultivation model in Southeast Guizhou. Guizhou Agric Sci 38(1):49-52

Wu Y, Huang M, Tao X, Guo T, Chen Z, Xiao W (2016) Quantitative trait loci identification and meta-analysis for rice panicle-related traits. Mol Genet Genomics 291(5):1-14

Yan QY (2008) A historic expedition of crops planting from glutinous rice to indicia rice in the Southeast Guizhou. Anc Mod Agric 3:27-34

Yu XQ, Jiang XH, Wu HB, Zhao DG, Fang XJ (2005) SSR genetic diversity of cold-tolerant native rice varieties in Guizhou, Southwest China. J Agri Sci 18(1):1-4

Zhao CF, Chen T, Zhao QY, Zhou LH, Zhao L, Zhang YD, Wang CL (2016) Analysis of QTLs for panicle exsertion and its relationship with yield and yield-related traits in rice (Oryza sativa L.). Genet Mol Res 15(2):gmr.15027423

Zheng XF, Fu Y, He XJ, Huang G, Yang AP, Yang LB (2010) Agronomic traits and classification of fragrant rice germplasm resource in Congjiang County. Seed 29(8):55-58

Zhou DH, Xu ZJ, Wang SL, Yang LJ (2003) A preliminary analysis of characters of rice panicles. J Shenyang Agric Univ 34(5):358-361

Zhou SM, Kang HX, Li QQ, Chen ZX, Zhang YF, Liu ED, Wang GL, Chen HQ, Pan XB (2014) Genome-wide association analysis on genes controlling panicle of varieties from International Rice Core Collection Bank and it breeding utilization and utilization of related genes of panicle traits in Rice. Chin J Rice Sci 28(6):649-658 